\title{
Dispersion Polymerization of Styrene Employing Lyophilic Comonomer in the Absence of Stabilizer: Synthesis of Impurity-free Microspheres
}

\author{
Hyekyung Han, Jeongwoo Lee, Jinho Hong, and Sang Eun Shim* \\ Department of Chemical Engineering, Inha University, Incheon 402-751, Korea
}

Received August 11, 2008; Revised December 17, 2008; Accepted December 19, 2008

\begin{abstract}
We investigated the feasibility of dispersion polymerization without any stabilizer, which has been considered essential for ensuring colloidal stability. By employing small amounts of a lyophilic comonomer, 4-vinyl pyridine, styrene was successfully polymerized by dispersion polymerization in aqueous alcohol without stabilizer to afford stable poly(styrene-co-4-vinyl pyridine) copolymer microspheres. The stable microspheres were produced in the 4-vinyl pyridine range of 2-15 wt $\%$ to styrene. Without 4-vinyl pyridine, severely coagulated particles were obtained, implying that the poly(4-vinyl pyridine) moiety endowed colloidal stability. The polymerization kinetics, behavior, and properties of the ultimate particles showed general features of dispersion polymerization. The study results suggest that stabilizer-free dispersion polymerization is possible, thereby facilitating the synthesis of impurity (stabilizer)-free polymer particles.
\end{abstract}

Keywords: poly(styrene-co-4-vinyl pyridine), stabilizer-free, dispersion polymerization, microspheres.

\section{Introduction}

Monodisperse polymeric microspheres have received much attention due to their vast application areas. ${ }^{1}$ Such microspheres have been used in information technology, chromatographic separation, electric and electronic application, and biotechnology. ${ }^{2-4}$ Recently, the monodisperse polymer particles have been applied to more advanced fields such as a template material for preparing three dimensionally ordered colloidal crystals. ${ }^{5}$ They are prepared by emulsion, dispersion, or suspension polymerizations. Among those techniques, dispersion polymerization has been an attractive alternative to prepare polymer microspheres, owing to its facility to obtain monodisperse microspheres with 1-10 $\mu \mathrm{m}$ size range in a single step. ${ }^{6}$ Also it is an attractive polymerization method due to relatively high conversion and fast process.

Dispersion polymerization consists of monomer, initiator, and steric stabilizer and all the components should be dissolved in the polymerization media such as alcohol and mixture of alcohol and water. ${ }^{7,8}$ Dispersion polymerization starts in a homogeneous phase, and then phase separation occurs when the nuclei are precipitated from the medium to give growing particles. Therefore, one of the criteria for choosing the components is solubility in the polymerization medium. ${ }^{9}$ In general, the steric stabilizer is an essential ingredient to produce stabilized particles with a spherical

*Corresponding Author. E-mail: seshim@inha.ac.kr shape by preventing the aggregation of colloidal particles in dispersion polymerization. But the steric stabilizer remains on the polymer particle surface after polymerization, either by physical adsorption or by chemical bond. The steric stabilizer brings out environmental pollution, cost increase of the product, change in the ultimate properties of polymer particles, and migration of stabilizer molecules. ${ }^{10}$

Therefore, Xing et al. ${ }^{10,11}$ recently reported a dispersion copolymerization for the preparation of surface-clean polymer particles without any stabilizer or other additives. They performed the novel stabilizer-free dispersion polymerization with maleic anhydride and vinyl acetate in alkyl esters as the reaction medium. The diameter of the copolymer particles is controlled in nanosize by changing the concentration of monomer or the ratio of monomers. The copolymer particles produced without any stabilizer or other additives are stabilized by the lyophilic property between the ester groups in both copolymer chains and reaction medium.

Homo-polymers and copolymers that contain a pyridine group such as poly(4-vinyl pyridine) (P4VP) have attracted significant interest due to various applications such as amphiphilic block copolymers and coordination reagents for metal nanoparticles. ${ }^{12-15}$ Especially, polymeric microspheres containing the pyridine group are currently utilized in the preparation of metal oxide-containing hybrid particles for electrophoretic ink, ${ }^{16} \mathrm{pH}$ and temperature responsive particles, ${ }^{17}$ support for catalysts, ${ }^{18}$ fabrication of electroluminescence devices, ${ }^{19}$ and the formation of core/inorganic shell structure. ${ }^{20}$ However, the synthesis of microspheres of styrene 
and 4-vinyl pyridine copolymers by dispersion polymerization in the absence of steric stabilizer has not been reported. In this study, the copolymerization of styrene with 4-vinyl pyridine was carried out using dispersion copolymerization in the absence of steric stabilizer to produce the impurity-free polymer particles. And the effects of various factors on the morphology of the copolymer microspheres were discussed in terms of the polymerization rate, molecular weight, diameter of particles and so on.

\section{Experimental}

Materials. Styrene (99\%, Junsei Chemicals, Japan) and 4-vinyl pyridine (99\%, Acros organics, Belgium) were purified using an inhibitor removal column (Aldrich, USA) and stored $-5{ }^{\circ} \mathrm{C}$ prior to use. As an initiator analytical grade of 2,2-azobis(isobutyronitrile) (AIBN, Junsei) was used without further purification. Ethanol (99\%, Samchun, Korea) and double-distilled deionized (DDI) water were used as the polymerization medium.

Polymerization Procedures. The dispersion copolymerization of styrene and 4-vinyl pyridine was conducted in a mixture of ethanol and DDI water with AIBN as an initiator. The amount of medium was controlled constant to be a volume ratio of ethanol/water $5 / 1(\mathrm{v} / \mathrm{v})$. When a high amount of water is used with ethanol as the medium, the size of ultimate particles decreases due to the difference of solubility parameters between the media and monomers. Therefore, the volume ratio of ethanol to water was fixed 5 to 1 in this study. $10 \mathrm{wt} \%$ of monomers (styrene and $4 \mathrm{VP}$ ) relative to the medium was charged and the concentration of 4VP was varied from 2 to $15 \mathrm{wt} \%$ relative to the amount of styrene monomer.

Dispersion copolymerization was carried out in a glass scintillation vial. First, $20 \mathrm{~mL}$ ethanol mixed with $4 \mathrm{~mL}$ DDI water was charged in the vial. The pre-weighed amounts of styrene and 4VP were added to the reaction vessel. Nitrogen was bubbled for $1 \mathrm{~h}$ in order to remove oxygen in the reactor. When the temperature of the reaction system was increased to $65{ }^{\circ} \mathrm{C}$, pre-weighed initiator was poured into the reactor and polymerization was carried out for $24 \mathrm{~h}$. After completion of polymerization, the latex was repeatedly washed with a mixed solution of ethanol and water and dried overnight in vacuo at $60{ }^{\circ} \mathrm{C}$.

Characterization. The conversion was calculated gravimetrically. Hitachi SEM (scanning electron microscopy) S-4300 was used to study the morphology of the P(St-co-4VP) particles. The number-average diameter $\left(D_{n}\right)$, the weight-average diameter $\left(D_{w}\right)$ and the coefficient of variation $\left(C_{V}\right)$ of particles diameter were defined using the following equations by counting at least 100 individual particles from SEM microphotographs.

$$
D_{n}=\left(\sum n_{i} d_{i} / n_{i}\right)
$$

$$
\begin{aligned}
& D_{w}=\left(\sum d_{i}^{3} / \Sigma d_{i}^{2}\right) \\
& \text { Uniformity }=\frac{D_{w}}{D_{n}} \\
& C_{V}=\frac{\left(\sum\left(d_{i}-\left(\sum n_{i} d_{i} / \Sigma n_{i}\right)\right)^{2} / \Sigma n_{i}\right)^{1 / 2}}{\left(\sum n_{i} d_{i} / \Sigma n_{i}\right)} \times 100
\end{aligned}
$$

where $n_{i}$ is the number of particles and $d_{i}$ is the diameter of particle $i$, uniformity is the ratio of the $D_{w}$ to $D_{n}$. The particle size distribution was also measured by a particle size analyzer (LS230 ${ }^{\circledR}$, Beckman Coulter).

The molecular weight and polydispersity index (PDI) were characterized using a Viscotek Gel Permeation Chromatography (GPC). Universal calibration curve was made using ten PS standard samples (Polymer Laboratories) with molecular weights of 580-7,500,000 g/mol. The P(St-co-4VP) copolymers dissolved in THF was injected at a flow rate of 1.0 $\mathrm{mL} / \mathrm{min}$. The glass transition temperature $\left(T_{g}\right)$ of the sample was measured by the Perkin-Elmer differential scanning calorimeter-7 (DSC). The sample was heated at the rate of $20{ }^{\circ} \mathrm{C} / \mathrm{min}$ under a nitrogen atmosphere and quench-cooled, and then reheated for a second run. The $T_{g}$ was determined at the deflection point in the transition region from the second scan. Measurement of the FTIR spectra was performed using a Bruker 48 series FTIR spectroscopy. All infrared spectra recorded at $4 \mathrm{~cm}^{-1} ; 21$ resolution and 16 scans were accumulated in $20 \mathrm{~s}$ for each specimen. The composition of the copolymer dissolved in $\mathrm{CDCl}_{3}$ was determined by using the ${ }^{1} \mathrm{H}$ NMR spectroscopy (UNITYINOVA 400, VARIAN, USA).

\section{Results and Discussion}

Effect of Agitation. Figure 1 shows the SEM photographs of $\mathrm{P}(\mathrm{St}-\mathrm{co}-4 \mathrm{VP})$ microspheres prepared by dispersion polymerization with $5 \mathrm{wt} \% 4 \mathrm{VP}$ relative to the amount of sty-
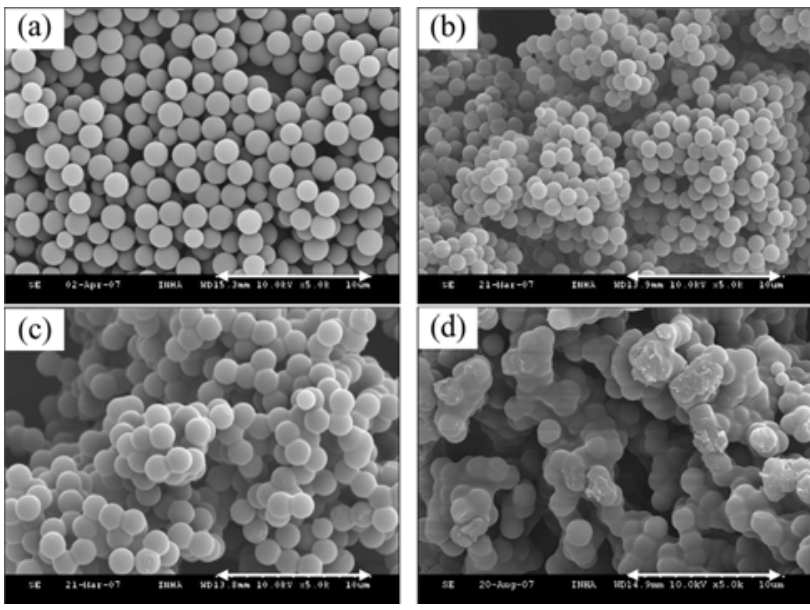

Figure 1. SEM microphotographs of $\mathrm{P}(\mathrm{St}-\mathrm{co}-4 \mathrm{VP})$ microspheres prepared at various agitation speeds; (a) $0 \mathrm{rpm}$, (b) $100 \mathrm{rpm}$, (c) $200 \mathrm{rpm}$, and (d) $300 \mathrm{rpm}$. Scale bar $=10 \mu \mathrm{m}$. 
Table I. Effect of Agitation Speed on the Formation of P(St-co4VP) Microspheres

\begin{tabular}{ccccc}
\hline rpm & $\begin{array}{c}\text { Primary Size } \\
(\mu \mathrm{m})\end{array}$ & Uniformity & $\begin{array}{c}C_{V} \\
(\%)\end{array}$ & $\begin{array}{c}\text { Conversion } \\
(\%)\end{array}$ \\
\hline 0 & $1.883^{a}(1.57)^{b}$ & 1.009 & $26.2(7.24)$ & 94.64 \\
100 & $12.32(1.07)$ & 1.004 & $37.98(4.69)$ & 87.86 \\
200 & $13.2(-)$ & 1.020 & $71.65(12.51)$ & 80.88 \\
\hline
\end{tabular}

${ }^{a}$ Size measurement using PSA. ${ }^{b}$ Size measurement using SEM image.

rene $(0.5 \mathrm{wt} \% 4 \mathrm{VP})$ at various agitation degrees $(0-300 \mathrm{rpm})$ in the absence of a steric stabilizer. It is seen that the copolymer microspheres having good colloidal stability can be formed in the absence of a stabilizer at $0 \mathrm{rpm}$. The dependence of average-size, the uniformity, coefficient of variation $\left(C_{V}\right)$ and the conversion of the polymerization with various agitation speeds are summarized in Table I. At $0 \mathrm{rpm}$, the particle size measured by a PSA is relatively close to the result obtained by SEM. However, at $100 \mathrm{rpm}$ and over, the data measured by a PSA are larger than SEM measurements because the polymer particles are partially coagulated. Table I shows that the particle size distributions are fairly monodisperse and well-defined at $0 \mathrm{rpm}$. And the conversion is higher than others. Therefore, it has been found that no agitation is the best to produce stable polymer microspheres in dispersion polymerization without a steric stabilizer and this condition was used in the all following experiments.

The mechanism of the formation of microspheres without any stabilizer would be quite similar to that of precipitation polymerization. Stover group has developed a method in which polymer particles are generated without coagulation in the precipitation polymerization without any auxiliary stabilizer. ${ }^{21-23}$ Also, we have synthesized various polymer particles using the same method and the relevant mechanism was speculated. ${ }^{24-26}$ In this specific case of precipitation polymerization, the colloidal stability of the microspheres is endowed by the fully crosslinked polymer chains at a very low agitation speed. Since no stabilizer is employed in this technique, the polymer particles lack stability against coagulation during the polymerization. Therefore, a high degree of crosslinking and low agitation speed are prerequisites for successful particle formation. However, alcohol soluble poly(4-vinyl pyridine) moiety provides steric stabilization in this study. In the beginning of the polymerization, vinyl pyridine and styrene are polymerized in the medium until two distinct oligomeric species undergo coupling copolymerization reaction. When the growing chains reach a certain critical limit length, the chains start to precipitate out of the medium by forming arbitrary primary particles. At this point, the homogeneous polymerization turns to heterogeneous system. At this stage of the formation of primary particles, lyophilic pyridine moiety with a better solubility in the medium tends to locate at the surface of the particles and they provide colloidal stability by steric hinderance which is

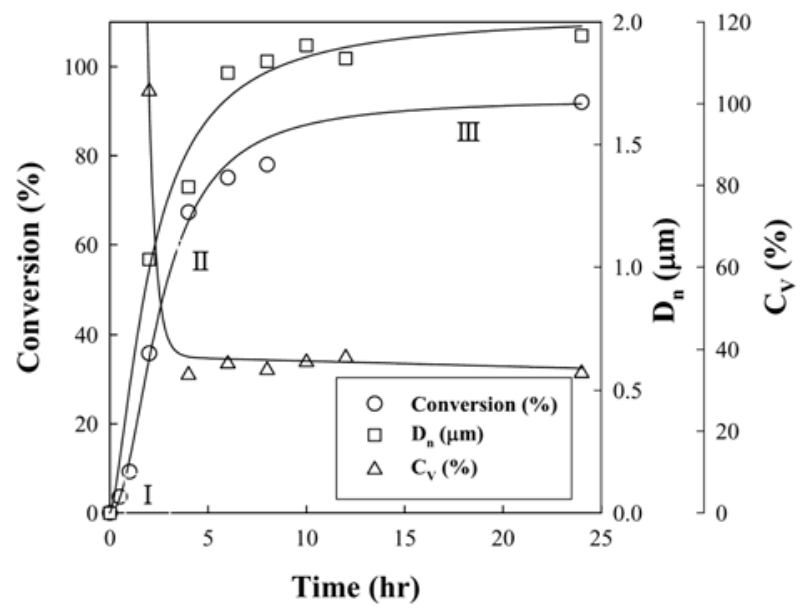

Figure 2. The conversion, $D_{n}$, and $C_{V}$ of $\mathrm{P}(\mathrm{St}-\mathrm{co}-4 \mathrm{VP})$ microspheres produced with $0.5 \mathrm{wt} \% 4 \mathrm{VP}$ prepared by dispersion polymerization without agitation at $65^{\circ} \mathrm{C}$.

commonly observed in dispersion polymerization in the presence of steric stabilizer. So stable microspheres can be successfully obtained without crosslinking as in precipitation polymerization. Since the primary particles formed without the aid of auxiliary stabilizer such as surfactant or steric stabilizer are vulnerable to the polymerization environment such as mechanical collision between the growing particles, a minimal shear force is needed during polymerization. Therefore, the best spherical morphology is achieved at zero rpm.

The polymerization results with $0.5 \mathrm{wt} \% 4 \mathrm{VP}$ under no agitation are depicted in Figure 2. The conversion of P(Stco-4VP) shows the typical behavior of the chain polymerization; gel effect. It is seen that the rate of polymerization was relatively low below $5 \%$ conversion at Stage I, while the conversion dramatically increased to $80 \%$ approximately after $6 \mathrm{~h}$ at Stage II, and reached a plateau at Stage III. In other words, the change of those steps means the change of the polymerization loci, namely the continuous and polymer phases. ${ }^{11}$ The lines in Figure 2 are achieved by curve-fitting the experimental data using a commercial software $\left(\mathrm{SigmaPlot}^{\mathbb{\mathbb { }}}\right)$.

As shown in Figure 2, the $\mathrm{P}(\mathrm{St}-\mathrm{co}-4 \mathrm{VP})$ microspheres continuously grow with the polymerization time, then become mature after $6 \mathrm{~h}$ of polymerization. In this plot, the number average diameter and the coefficient of variation of particle size were measured by Coulter LS $230^{\circledR}$. The coefficient of variation shows the typical behavior of the heterogeneous polymerization. $^{27}$

The molecular weight evolution and polydispersity index (PDI) of P(St-co-4VP) copolymers with the conversion are given in Figure 3. Above $70 \%$ of conversion, the average molecular weight decrease and PDI increase as increasing conversion. It means that monomer and initiator concentration become low in particles and then the polymerization loci shift to continuous phase, which causes a decrease in $M_{w}$ and increase in PDI. 


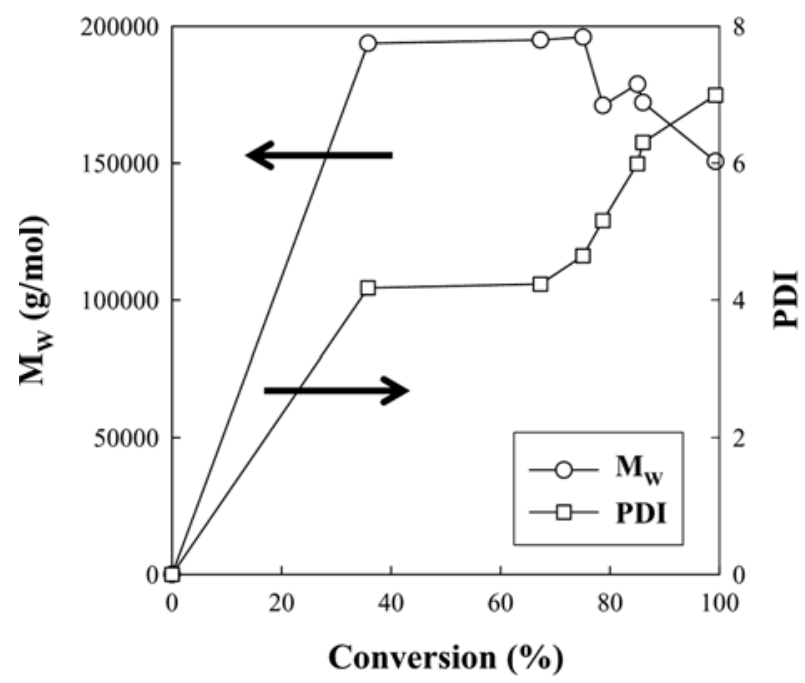

Figure 3. Evolution of $M_{w}$ and PDI of poly(St-co-4VP) microspheres prepared with $0.5 \mathrm{wt} \% 4 \mathrm{VP}$ by dispersion polymerization with no agitation $65^{\circ} \mathrm{C}$.
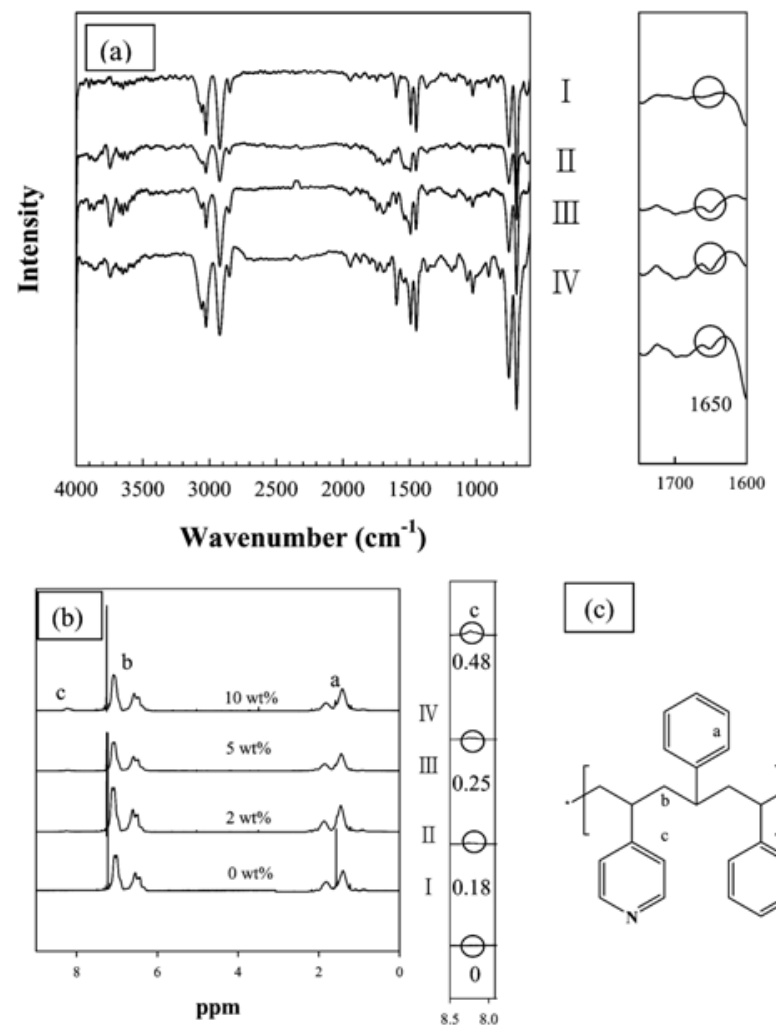

(c)

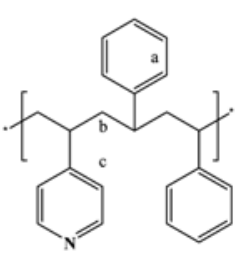

Figure 4. (a) FTIR spectra, (b) NMR spectra of $\mathrm{P}(\mathrm{St}-\mathrm{co}-4 \mathrm{VP})$ copolymer with different concentrations of 4VP; (I) 0; PS homopolymer (II) 2, (III) 5, and (IV) $10 \mathrm{wt} \%$ relative to styrene, and (c) chemical structure of $\mathrm{P}(\mathrm{St}-\mathrm{co}-4 \mathrm{VP})$.

The chemical compositions of the copolymer microspheres prepared with an increasing amount of 4VP were characterized by FTIR and ${ }^{1} \mathrm{H}$ NMR spectroscopies, as shown in Figures 4(a) and (b), respectively. In the FTIR spectra (Figure 4(a)), the spectrum of homo-PS is similar to that of the $\mathrm{P}(\mathrm{St}-$ co-4VP) except for a peak of $\mathrm{C}=\mathrm{N}$ at $1690-1630 \mathrm{~cm}^{-1}$ (spectra II, III, IV). The characteristic peak at $1690-1630 \mathrm{~cm}^{-1}$ suggests that the polymer microspheres prepared by dispersion polymerization consist of St/4VP copolymer. The ${ }^{1} \mathrm{H}$ NMR spectroscopy provides further insight to the copolymerization of styrene with 4VP. Figure 4(b) shows the ${ }^{1} \mathrm{H}$ NMR spectra of both PS and P(St-co-4VP) and the peaks 'c' (spectra II, III, IV) at $8-8.5 \mathrm{ppm}$ are due to two protons in the meta-position of the pyridine ring. ${ }^{28}$ With increasing amount of 4VP from 0 to $10 \mathrm{wt} \%$, the ratio of the peak area at 8-8.5 ppm to the characteristics peak of polystyrene at $1.82 \mathrm{ppm}$ increases from $0 \%$ at $0 \mathrm{wt} \%, 8.25 \%$ at $2 \mathrm{wt} \%$, $11.68 \%$ at $5 \mathrm{wt} \%$, and $23.88 \%$ at $10 \mathrm{wt} \%$, respectively. These peaks indicate that $\mathrm{P}(\mathrm{St}-\mathrm{co}-4 \mathrm{VP})$ was formed successfully.

Effect of 4VP Concentration to Styrene. The 4VP concentration relative to styrene is important because major characteristics are determined by 4VP. Figure 5 shows the SEM microphotographs of PS homopolymer and P(St-co-4VP) copolymer particles prepared with various $4 \mathrm{VP}$ concentrations; 2, 5, 10, and $15 \mathrm{wt} \%$ relative to styrene. Figure 5(a) represents the morphology of the final PS particles without a steric stabilizer. Without $4 \mathrm{VP}$, non-stabilized particles with coagulation are formed and moreover conversion is low $57.23 \%$ as shown in Table II. At $15 \mathrm{wt} \%$ 4VP, the coagulated particles were again observed since long P4VP moiety has a lyophilic nature. Therefore, $4 \mathrm{VP}$ below $15 \mathrm{wt} \%$ is essential to stabilize the particles in the absence of a steric
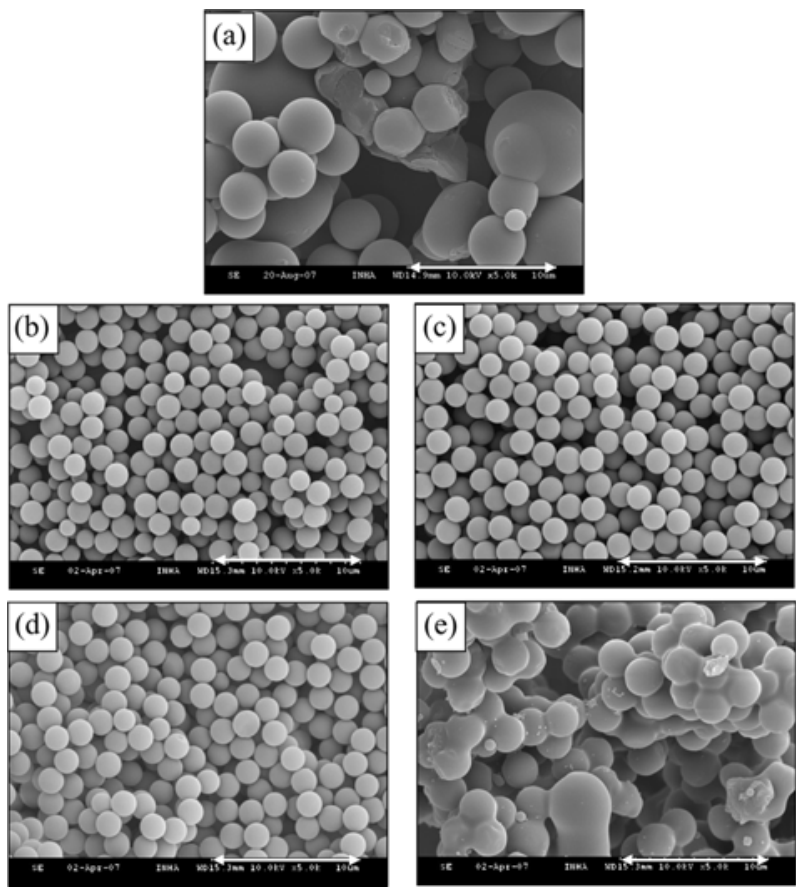

Figure 5. SEM microphotographs of $\mathrm{P}(\mathrm{St}-\mathrm{co}-4 \mathrm{VP})$ microspheres with various concentrations of 4VP; (a) 0 (PS microspheres), (b) 2 , (c) 5 , (d) 10 , and (e) $15 \mathrm{wt} \%$ relative to the amount of styrene. Scale bar $=10 \mu \mathrm{m}$. 
Table II. Effect of $4 \mathrm{VP}$ Concentration on the Formation of P(Stco-4VP) Microspheres

\begin{tabular}{ccccc}
\hline $\begin{array}{c}4 \mathrm{VP} \\
(\mathrm{wt} \%)\end{array}$ & $\begin{array}{c}\text { Primary Size } \\
(\mu \mathrm{m})\end{array}$ & Uniformity & $\begin{array}{c}C_{V} \\
(\%)\end{array}$ & $\begin{array}{c}\text { Conversion } \\
(\%)\end{array}$ \\
\hline 0 & 2.88 & 1.180 & 34.48 & 57.13 \\
2 & 1.57 & 1.007 & 6.11 & 88.02 \\
5 & 1.64 & 1.009 & 7.24 & 99.64 \\
10 & 1.69 & 1.002 & 3.52 & 99.75 \\
15 & 20.76 & 1.030 & 45.56 & 87.86 \\
\hline
\end{tabular}

stabilizer. By excluding the extremely coagulated cases at 0 and $15 \mathrm{wt} \% 4 \mathrm{VP}$, the average diameter of the particles tends to slightly increase from 1.57 to $1.69 \mu \mathrm{m}$ with the corresponding increase in the amount of 4VP from 2 to $10 \mathrm{wt} \%$. Since the 4VP has a good solubility in the medium, more oligomeric chains are dissolved at a high concentration of 4VP, implying that the number of primary particles is reduced. In turn, this means that the amount of reactive monomer or oligomer per a primary particle is greater, finally resulting in the larger sized ultimate particles.

To investigate the copolymer chain structure, the glass transition temperature $\left(T_{g}\right)$ may provide useful clues about the structure of copolymers. The $T_{g}$ was measured by the differential scanning calorimetry (DSC) from 20 to $250^{\circ} \mathrm{C}$ at $20^{\circ} \mathrm{C} / \mathrm{min}$, and their values are plotted in Figure 6(a). The $T_{g}$ of the $\mathrm{P}(\mathrm{St}-c o-4 \mathrm{VP})$ copolymers were prepared with various concentrations of $4 \mathrm{VP}$. The $T_{g}$ of PS is $100^{\circ} \mathrm{C}$ and that of P4VP is $142^{\circ} \mathrm{C}^{29}$ The corresponding values of $T_{g}$ were calculated using the following Fox equation:

$$
\frac{1}{T_{g}}=\frac{w_{1}}{T_{g 1}}+\frac{w_{2}}{T_{g 2}}
$$

where $w_{1}$ and $T_{g 1}$ correspond to weight fraction and glass transition temperature of PS, and $w_{2}$ and $T_{g 2}$ the weight fraction and glass transition of P4VP, respectively. Theoretical and experimental values for each $\mathrm{P}(\mathrm{St}-\mathrm{co}-4 \mathrm{VP})$ are compared in Figure 6(b). The results from DSC indicate that all synthesized copolymers having only one glass transition ranged from 103 to $110^{\circ} \mathrm{C}$ and experimental values show a good agreement with theoretical ones, indicating that all copolymer chains prepared by dispersion polymerization are random and homogeneous.

The $M_{w}$ and PDI of P(St-co-4VP) copolymers with the concentration of 4VP monomer are given in Figure 7. The molecular weight decreased with increasing 4VP concentration. As shown in Figure 5, the average diameter of the particles increases with the 4VP amount. It is typically observed that there is the inverse relationship between the molecular weight and the size of the polymer particles in the dispersion polymerization. ${ }^{30}$ This phenomenon is ascribed to the suppressed termination reaction in particle phase due to the high viscosity of the monomer/polymer particle medium,
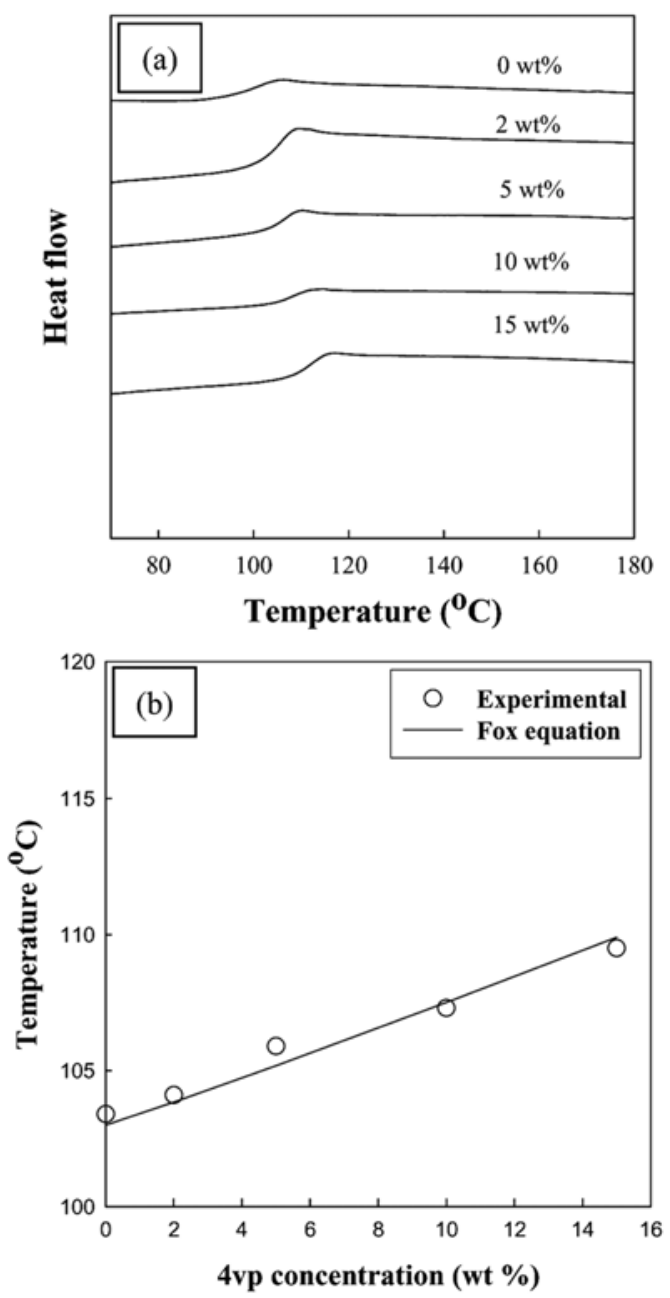

Figure 6. (a) DSC thermograms and (b) theoretical and experimental $T_{g}$ values of $\mathrm{P}(\mathrm{St}-\mathrm{co}-4 \mathrm{VP})$ copolymer by dispersion polymerization with various concentrations of $4 \mathrm{VP}$.

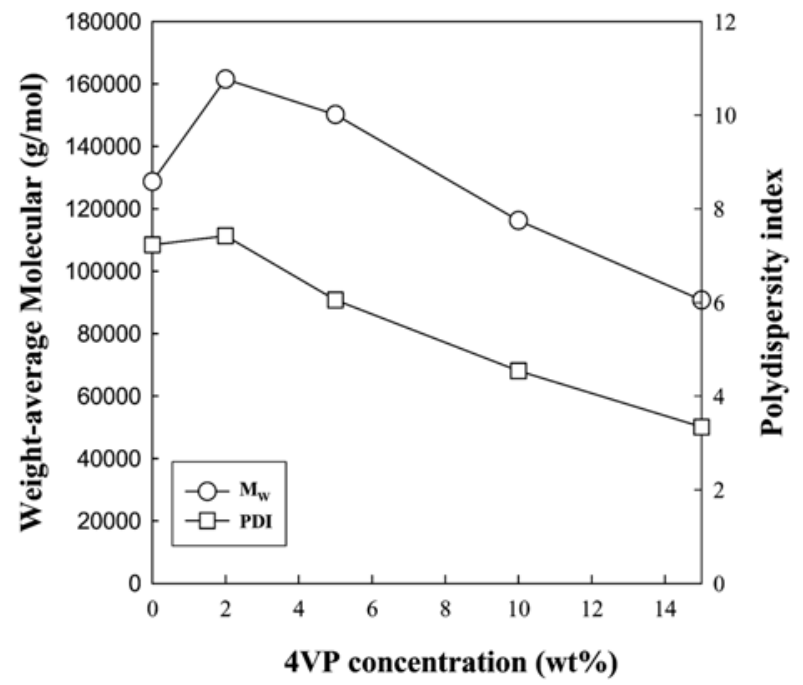

Figure 7. $M_{w}$ and $\mathrm{PDI}$ of $\mathrm{P}(\mathrm{St}-\mathrm{co}-\mathrm{VPP})$ microspheres prepared by dispersion polymerization with various concentrations of $4 \mathrm{VP}$. 
which more significantly occurs in small particles. Furthermore, the oligomer capture more readily occurs for small particles due to their greater surface area, which gives higher molecular weight. Finally, the smaller-sized particles give the higher molecular weight.

Effect of Reaction Temperature. Since $0.5 \mathrm{wt} \%$ 4VP and $0 \mathrm{rpm}$ resulted in the most stabilized particles, the concentration of 4VP and agitation were also fixed while the reaction temperature increased in this section. The reaction temperature was increased by $10^{\circ} \mathrm{C}$ increment from 55 to $75^{\circ} \mathrm{C}$. In all cases, well-defined microspheres are obtained. Consequently, the particle size and particle size distribution were increased with increasing the reaction temperature.

The SEM microphotographs of the $\mathrm{P}(\mathrm{St}-\mathrm{co}-4 \mathrm{VP})$ particles synthesized at each temperature are presented in Figure 8. It is seen that the size of $\mathrm{P}(\mathrm{St}-\mathrm{co}-4 \mathrm{VP})$ microspheres increases as increasing temperature. It is the typical behavior of dispersion polymerization. These phenomena have been explained by the fact that the increased temperature produces the greater number of active radicals, resulting in accelerating
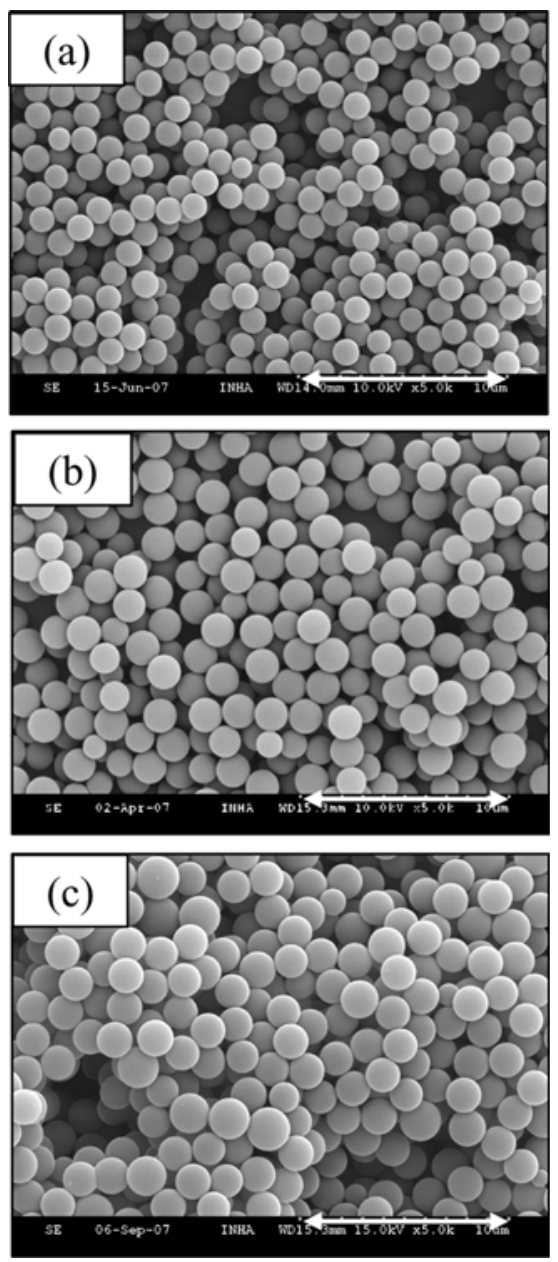

Figure 8. SEM microphotographs of $\mathrm{P}(\mathrm{St}-\mathrm{co}-4 \mathrm{VP})$ microspheres prepared with $5 \mathrm{wt} \%$ of $4 \mathrm{VP}$ at various temperatures; (a) $55^{\circ} \mathrm{C}$, (b) $65^{\circ} \mathrm{C}$, and (c) $75^{\circ} \mathrm{C}$. Scale bar $=10 \mu \mathrm{m}$.
Table III. Effect of the Temperature on the Formation of P(St-co-4VP) Microspheres

\begin{tabular}{ccccc}
\hline $\begin{array}{c}\text { Temp. } \\
\left({ }^{\circ} \mathrm{C}\right)\end{array}$ & $\begin{array}{c}\text { Primary Size } \\
(\mu \mathrm{m})\end{array}$ & Uniformity & $\begin{array}{c}C_{V} \\
(\%)\end{array}$ & $\begin{array}{c}\text { Conversion } \\
(\%)\end{array}$ \\
\hline 55 & 1.24 & 1.079 & 6.47 & 93.17 \\
65 & 1.57 & 1.009 & 7.24 & 94.64 \\
75 & 1.68 & 1.011 & 7.53 & 92.39 \\
\hline
\end{tabular}

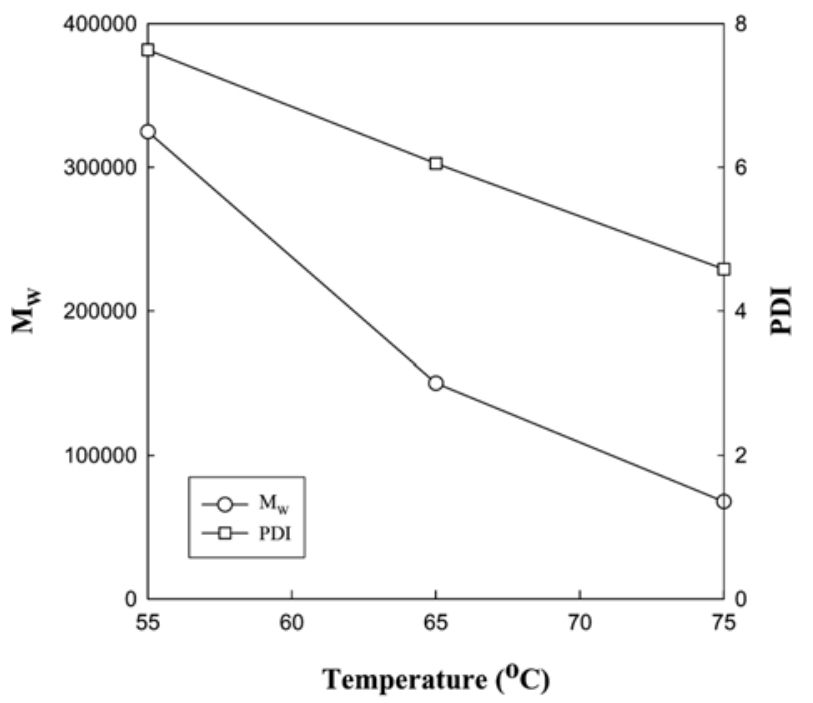

Figure 9. $M_{w}$ and $\mathrm{PDI}$ of $\mathrm{P}(\mathrm{St}-\mathrm{co}-4 \mathrm{VP})$ microspheres prepared with $5 \mathrm{wt} \%$ of $4 \mathrm{VP}$ by dispersion polymerization at various temperatures.

the aggregation of nuclei and followed by a formation of larger particles. ${ }^{9,31,32}$ The results are listed in Table III. And the $M_{w}$ and PDI are represented in Figure 9. It is seen that as the reaction temperature is increased, the greater number of active radicals is produced and then the primary nuclei in the early stage are increased. Therefore, the $M_{w}$ and PDI are decreased at a high temperature.

In addition, the high temperature reduces the viscosity of the polymerization medium while increases in polymerization and termination rate. ${ }^{33}$ Figure 10 shows the rate of polymerization $\left(R_{p}\right)$ calculated by taking the first-derivative of each conversion curve. The $R_{p}$ initially increased with increasing time due to the nucleation, and then reached a maximum. After that, the $R_{p}$ decreased with time. This trend is a characteristic in radical polymerization. The higher the reaction temperature, the faster the initiation step. Therefore, the $R_{p}$ increased with increasing temperature. The maximum rate of polymerization are $0.5290 \mathrm{~mol} / \mathrm{Ls}$ at $75^{\circ} \mathrm{C}, 0.4244 \mathrm{~mol} / \mathrm{Ls}$ at $65^{\circ} \mathrm{C}$ and $0.3210 \mathrm{~mol} / \mathrm{Ls}$ at $55^{\circ} \mathrm{C}$, respectively.

\section{Conclusions}

In this study, we confired the possibility of the synthesis 


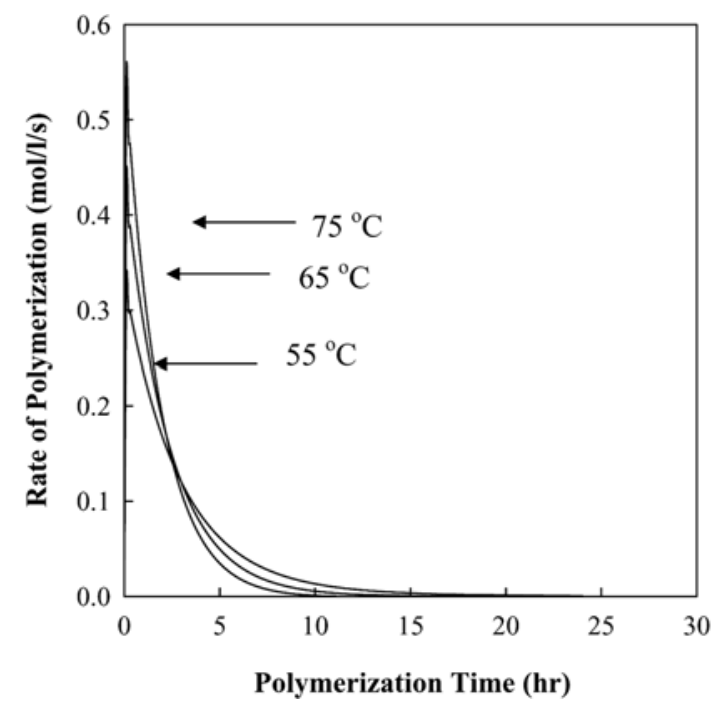

Figure 10. The effect of temperature on the rate of dispersion copolymerization of St and 4VP in the absence of steric stabilizer.

of stable polymeric microspheres by dispersion polymerization in the absence of steric stabilizer which has been considered to be an essential ingredient to stabilize resulting polymer particles. By employing small amounts of lyophilic comonomer, 4VP, styrene was successfully polymerized by dispersion polymerization in aqueous alcohol without stabilizer to give stable $\mathrm{P}(\mathrm{St}-\mathrm{co}-4 \mathrm{VP})$ copolymer microspheres where P4VP moiety plays a pivotal role to stabilize particles at stationary conditions, i.e. no agitation. The formation of the particles takes place in a very similar manner to precipitation polymerization however crosslinking is not necessary to provide colloidal stability herein. The polymerization kinetics, behavior, and the properties of the ultimate particles show general features of dispersion polymerization. The stable microspheres are produced only in the 4VP range of $2-15 \mathrm{wt} \%$ to styrene. Without 4VP, severely coagulated particles are obtained, implying that P4VP moiety endows colloidal stability. The spectroscopy and thermal properties indicate that the formed polymeric chains are random and homogenous $\mathrm{P}(\mathrm{St}-\mathrm{co}-$ 4VP). Finally, this study suggests that stabilizer-free dispersion polymerization is possible so that one can synthesize the impurity (stabilizer)-free polymer particles.

Acknowledgement. This work was supported by Inha University Research Grant.

\section{References}

(1) S. E. Shim, S. Yang, H. Jung, and S. Choe, Macromol. Res., 12, 233 (2004).

(2) H. Fudouz and Y. Xia, Adv. Mater., 15, 892 (2003).

(3) J. Ugelstad, P. Stenstad, L. Kilaas, W. S. Prestvik, A. Rian, K. Nustad, R. Herje, and A. Berge, Macromol. Symp., 101, 491
(1996).

(4) V. L. Covolan, L. H. I. Mei, and C. L. Mei, Polym. Adv. Technol., 8, 44 (1997).

(5) S. O. Cho, H. Y. Jun, and S. K. Ahn, Adv. Mater., 17, 120 (2005).

(6) J. Hong, C. K. Hong, and S. E. Shim, Colloid Surf. A, 302, 225 (2007).

(7) K. C. Lee and S. Y. Lee, Macromol. Res., 15, 255 (2007).

(8) K. C. Lee and S. Y. Lee, Macromol. Res., 16, 293 (2008).

(9) J. Lee, J. U. Ha, S. Choe, C. Lee, and S. E. Shim, J. Colloid Interf. Sci., 298, 663 (2006).

(10) C. Xing and W. J. Yang, Polym. Sci. Part A: Polym. Chem., 43, 3760 (2005).

(11) C. Xing, Y. Yu, and W. J. Yang, Macromol. Chem. Phys., 207, 621 (2006).

(12) S. Park, W. Sul, and Y. Chang, Macromolecules, 40, 3757 (2007).

(13) J. M. Lee, B. H. Lee, and S. Choe, Polymer, 47, 3838 (2006).

(14) H. Dong, E. Fey, A. Gandelman, and W. E. Jones, Jr., Chem. Mater, 18, 2008 (2006).

(15) P. L. Soo, S. N. Sidorov, J. Mui, L. M. Bronstein, H. Vali, A. Eisenberg, and D. Maysinger, Langmuir, 23, 4830 (2007).

(16) M. Badila, C. Brochon, A. Hebraud, and G. Hadziioannou, Polymer, 49, 4529 (2008).

(17) K. S. Kim and B. Vincent, Polym. J., 37, 565 (2005).

(18) W. T. Ford and P. D. Paul, Microsphere Microcap. Liposome., 4, 171 (2002).

(19) Q. Chen, X. Shen, and H. Gao, Colloid Surf. A, 275, 45 (2006).

(20) S. Pathak, M. T. Greci, R. C. Kwong, K. Mercado, G. K. S. Prakash, A. George, and M. E. Thompson, Chem. Mater., 12, 1985 (2000).

(21) W. H. Li and H. D. H. Stover, J. Polym. Sci. Part A: Polym. Chem., 37, 2899 (1999).

(22) J. S. Downey, G. McIsaac, R. S. Frank, and H. D. H. Stover, Macromolecules, 34, 4534 (2001).

(23) W. H. Li and H. D. H. Stover, J. Polym. Sci. Part A: Polym. Chem., 36, 1543 (1998).

(24) S. E. Shim, S. Yang, H. H. Choi, and S. Choe, J. Polym. Sci. Part A: Polym. Chem., 42, 835 (2004).

(25) S. E. Shim, S. Yang, M. J. Jin, Y. H. Chang, and S. Choe, Colloid Polym. Sci., 283, 41 (2004).

(26) S. E. Shim, S. Yang, and S. Choe, J. Polym. Sci. Polym. Chem., 42, 3967 (2004).

(27) R. G. Gilbert, Emulsion Polymerization: A Mechanical Approach, Academic Press, London, 1995.

(28) V. D. Mochel, Meeting of the Division of Rubber Chemistry, American Chemical Society, Montreal, Canada, 1967.

(29) M. Alger, Polymr Science Dictionary, $2^{\text {nd }}$ ed., Chapman \& Hall, London, 1997.

(30) A. J. Pains, W. Luymes, and J. McNulty, Macromolecules, 23, 3104 (1990).

(31) C. M. Tseng, Y. Y. Lu, and M. S. El-Aasser, J. Polym. Sci. Polym. Chem. Ed., 24, 2995 (1986).

(32) S. Shen, E. D. Sudol, and M. S. El-Aasser, J. Polym. Sci. Polym. Chem. Ed., 31, 1393 (1993).

(33) S. Kiatkamjornwong and C. Kongsupapsiri, Polym. Int., 49, 1395 (2000). 